\title{
His-Purkinje System Pacing Upgrade Improve the Heart Performances in Patients Suffering from Pacing-induced Cardiomyopathy with or without Permanent Atrial Fibrillation
}

\author{
Yiheng Yang $^{1}$, kexin wang ${ }^{1}$, peipei $\mathrm{Ma}^{1}$, rongfeng zhang ${ }^{1}$, Bin Waleed Khalid ${ }^{1}$, Xiaomeng \\ Yin ${ }^{1}$, Lianjun $\mathrm{Gao}^{1}$, Yunlong Xia ${ }^{1}$, and yingxue Dong ${ }^{1}$ \\ ${ }^{1}$ First Affiliated Hospital of Dalian Medical University
}

October 21, 2020

\begin{abstract}
The efficacy and safety of His bundle pacing (HBP) and left bundle branch pacing (LBBP) upgrades in patients with pacinginduced cardiomyopathy (PICM) and atrial fibrillation (AF) are still unknown. Methods and results Patients with PICM were continuous enrolled from January 2018 to March 2020. All patients were further divided into AF subgroup and sinus rhythm subgroup. Clinical data including echocardiographic examination parameters, electrocardiogram (ECG) measurements, and New York Heart Association (NYHA) classification, were assessed before and after a his-purkinje system pacing (HPSP) upgrade. The HBP and LBBP upgrades were completed in 34 of 36 (94\%), Complications including electrode dislodged, perforation, infection or thrombosis were not observed in perioperative period. During a mean of $11.52 \pm 5.40$ months of followup. The left ventricular ejection fraction (LVEF) increased significantly (33.76 \pm 7.54 vs $40.41 \pm 9.06, \mathrm{P}<0.001)$, and the QRS duration decreased $(184.22 \pm 23.76 \mathrm{~ms}$ vs $120.52 \pm 16.67 \mathrm{~ms}, \mathrm{P}<0.001)$ after the HBP upgrades. LVEDD reversed from $59.29 \pm 7.74$ $\mathrm{mm}$ to $53.91 \pm 5.92 \mathrm{~mm}(\mathrm{P}<0.001)$, and the NYHA functional class also improved to $2.00 \pm 0.76$ from $2.55 \pm 0.91$ at the first followup $(\mathrm{P}<0.001)$. The left atrium (LA) size also slightly decreased compared to the initial state $(59.29 \pm 7.74 \mathrm{~mm} \mathrm{VS} 56.44 \pm 6.46$, $\mathrm{P}=0.005)$. The threshold o did not increase significantly (1.18 $\pm 0.76 \mathrm{mv} @ 0.4 \mathrm{~ms}$ vs $1.26 \pm 0.91 \mathrm{mv} @ 0.4 \mathrm{~ms}, \mathrm{P}=0.581)$. These improvements in patients with AF were similar with those in patients without AF $(\mathrm{P}>0.05)$. Conclusion HBP and LBBP upgrades improved the heart performance and reversed the left ventricular remodeling in patients suffering from PICM with or without $\mathrm{AF}$, and it should be a promising choice in these patients.
\end{abstract}

\section{Introduction}

It is well known that long-term right ventricular paving (RVP) might lead to a long QRS duration (QRSd) and left ventricular (LV) dyssynchrony and consequently result in LV systolic dysfunction. And more and more studies have demonstrated that conventional pacing sites (i.e., the apex or septum) could increase mortality and hospitalization of heart failure in pacemaker-dependent patients [1-3]. However, the incidence of PICM remains relatively high, and no response to cardiac resynchronization therapy (CRT) for these patients. And the biventricular pacing upgrade is still not the optimal recommendation (IIb) for these patients in recent guidelines [4].

What would help those patients with PICM? His-Purkinje system pacing, including HBP and LBBP, was chosen as an alternative procedure in patients with indications of bradycardia or heart failure [4]. The safety and efficiency have been confirmed by recent publications [5-7]. However, studies focusing on the outcome of HBP and LBBP upgrades in PICM patients are extremely rare $[8,9]$. It is unknown whether this is an effective procedure in patients with PICM and AF. We consequently performed this study to investigate the clinical outcome of HBP and LBBP upgrades in these patients. 


\section{Methods and results}

Patients with PICM were continuous enrolled from January 2018 to March 2020. All patients were further divided into AF subgroup and sinus rhythm subgroup. Clinical data including echocardiographic examination parameters, electrocardiogram (ECG) measurements, and New York Heart Association (NYHA) classification, were assessed before and after a his-purkinje system pacing (HPSP) upgrade. The HBP and LBBP upgrades were completed in 34 of 36 (94\%), Complications including electrode dislodged, perforation, infection or thrombosis were not observed in perioperative period. During a mean of $11.52 \pm 5.40$ months of follow-up. The left ventricular ejection fraction (LVEF) increased significantly (33.76 \pm 7.54 vs $40.41 \pm 9.06$, $\mathrm{P}<0.001)$, and the QRS duration decreased $(184.22 \pm 23.76 \mathrm{~ms}$ vs $120.52 \pm 16.67 \mathrm{~ms}, \mathrm{P}<0.001)$ after the HBP upgrades. LVEDD reversed from $59.29 \pm 7.74 \mathrm{~mm}$ to $53.91 \pm 5.92 \mathrm{~mm}(\mathrm{P}<0.001)$, and the NYHA functional class also improved to $2.00 \pm 0.76$ from $2.55 \pm 0.91$ at the first follow-up $(\mathrm{P}<0.001)$. The left atrium (LA) size also slightly decreased compared to the initial state $(47.44 \pm 7.14 \mathrm{~mm}$ VS $45.56 \pm 7.78, \mathrm{P}=0.010)$. The threshold did not increase significantly (1.18 \pm 0.76 mv@0.4ms vs $1.26 \pm 0.91 \mathrm{mv} @ 0.4 \mathrm{~ms}, \mathrm{P}=0.581)$. These improvements in patients with $\mathrm{AF}$ were similar with those in patients without $\mathrm{AF}(\mathrm{P}>0.05)$.

\section{Conclusions}

HBP and LBBP upgrades improved the heart performance and reversed the left ventricular remodeling in patients suffering from PICM with or without AF, and it should be a promising choice in these patients.

Keywords: His-Purkinje System Pacing, Pacing-induced cardiomyopathy, Cardiac function

\section{Introduction}

It is well known that long-term right ventricular paving (RVP) might lead to a long QRS duration (QRSd) and left ventricular (LV) dyssynchrony and consequently result in LV systolic dysfunction. And more and more studies have demonstrated that conventional pacing sites (i.e., the apex or septum) could increase mortality and hospitalization of heart failure in pacemaker-dependent patients [1-3]. However, the incidence of PICM remains relatively high, and no response to cardiac resynchronization therapy (CRT) for these patients. And the biventricular pacing upgrade is still not the optimal recommendation (IIb) for these patients in recent guidelines [4].

What would help those patients with PICM? His-Purkinje system pacing, including HBP and LBBP, was chosen as an alternative procedure in patients with indications of bradycardia or heart failure [4]. The safety and efficiency have been confirmed by recent publications [5-7]. However, studies focusing on the outcome of HBP and LBBP upgrades in PICM patients are extremely rare [8,9]. It is unknown whether this is an effective procedure in patients with PICM and AF. We consequently performed this study to investigate the clinical outcome of HBP and LBBP upgrades in these patients.

\section{Methods}

\section{Patients enrollment}

Patients with PICM were continuous enrolled from January 2018 to January 2020 in the first affiliated hospital of Dalian Medical University. The clinical data, including echocardiographic examination parameters, ECG measurements, and NYHA classification, were assessed before and after an HBP upgrade.

PICM was defined as RVP dependence (RVP $i 40 \%$ ) and new onset LVEF decreases $>10 \%$ from baseline resulting in an LVEF of [?] $40 \%$ without other identifiable causes. Patients with other identifiable causes of heart failure, including myocardial infarction, severe valvular heart disease, arrhythmia-related cardiomyopathy, and long-term uncontrolled hypertension were excluded. Permanent AF refers to AF in which cardioversion has failed and $\mathrm{AF}$ that has been sustained for more than 1 year.

\section{Upgrade procedure}

The 4.1F 3830 (SelectSecure, Medtronic, Minneapolis, MN) pacing lead was delivered using the C315HIS (Medtronic, Minneapolis, MN) sheath. His bundle electrograms were mapped in a unipolar configuration 
and recorded on the recording system (Prucka Cardiolab, GE Healthcare, Waukesha, WI). To locate the optimal site for the His bundle lead, the sheathes and leads were delivered to the ventricular end for the distal HBP/LBBP, and the pacing rate was decreased to $30 \mathrm{bpm}$ for an escape rhythm. If no his electrogram was observed, pace mapping was conducted to identify a site with evidence for His bundle capture. His bundle pacing was acceptable when capture threshold was lower than $2.0 \mathrm{~V} / 1.0 \mathrm{~ms}$.For the patients with third-degree atrioventricular block (IIIdegAVB) or ventricular pacing dependent, right ventricular backup pacing was retained if threshold of his bundle pacing is higher than $2.0 \mathrm{v} / 0.5 \mathrm{~ms}$. LBBP would be further performed when HBP failed.

\section{Follow-up}

Patients were followed up in the cardiology and device clinic and at 1, 3, 6, 12, 18 and 24 months. Clinical data, including the NYHA class, QRS duration, device programming information and echocardiography parameters, were recorded.

\section{Statistical analysis}

Continuous variables are expressed as the mean +- SD (standard deviation) and were compared with paired $\mathrm{t}$ tests for normally distributed data. Categorical variables are expressed as percentages $(\%)$ and were compared using $\chi^{2}$ tests. Nonparametric tests were used if the data were not normally distributed. All statistical tests were two-tailed; $\mathrm{P}<0.05$ was considered to indicate statistical significance.

\section{Results}

\section{Baseline characteristics}

Permanent HBP or LBBP was successful in 34 of 36 patients. The average LVEF value was dramatically low (33.76 $\pm 7.54 \%$ vs. $51.77 \pm 8.19 \%)$ after right ventricular septal pacing for 79.18 (19-321) months, and 12 patients showed elective replacement indicators (ERIs) at the latest programming before the upgrade procedure. Permanent HBP upgrades were successful in 29 of 34 patients, and LBBP upgrades were successful in 5 patients. Two patients failed in upgrades, and one of them died for heart failure after 12 months follow-up. Eight patients were permanent AF. Nine patients (26.47\%) were infranodal block. The devices of CRT were implanted in $13(38.24 \%)$ patients. Dual-chamber pacemakers were implanted in 20 patients $(58.82 \%)$, and implantable cardioverter defibrillator (ICD) were implanted in one patient (2.94\%). The baseline characteristics were shown in Table 1 .

\section{Clinical outcomes of upgrades}

The clinical outcomes before and after upgrades were shown in Figure 1. After upgrades, the LVEF significantly increased from baseline $33.76 \pm 7.54 \%$ to $40.41 \pm 9.06 \%(\mathrm{P}<0.001)$ (Figure 1A). The paced QRSd markedly decreased from $184.2 \pm 23.76 \mathrm{~ms}$ at baseline to $120.5 \pm 16.67 \mathrm{~ms}$ after HPSP $(\mathrm{P}<0.001)$ (Figure 1B). The LVEDD reversed from $59.29 \pm 7.74 \mathrm{~mm}$ to $53.91 \pm 5.92 \mathrm{~mm}(\mathrm{P}<0.001)$ (Figure $1 \mathrm{C})$. The NYHA functional class improved to $2.00 \pm 0.76$ from $2.55 \pm 0.91$ at baseline during follow-up $(\mathrm{P}<0.001)$.

\section{Clinical outcomes of upgrades in patients with permanent AF}

Twenty-one patients were permanent AF. These improvements of cardiac function and remodeling also were observed in patients with permanent AF after upgrades (Table 2). To note, left atrium (LA) size also slightly decreased from $59.29 \pm 7.74 \mathrm{~mm}$ at baseline to $56.44 \pm 6.46$ after HPSP $(\mathrm{P}=0.010)$. No significant difference was found in mitral $(10 / 6, \mathrm{P}=0.219)$ or tricuspid regurgitation $(10 / 8, \mathrm{P}=0.727)$ before and after HPSP.

There was no significant difference in the improvement of the LVEF between the patients with or without AF $(\mathrm{P}=0.424)$. The improvements of LVEDD, QRSd also had no significant difference between the two subgroups.

\section{Lead outcome of upgrades}


The pacemaker parameters including threshold, sensed $\mathrm{R}$ wave and impedance were recorded after a median follow-up period of 8.9 months (Table 3). The threshold of HPSP did not increased significantly (1.18 \pm 0.76 mv@0.4ms vs $1.26 \pm 0.70 \mathrm{mv} @ 0.4 \mathrm{~ms}, \mathrm{P}=0.581)$ (Figure 1D). Sensed R (4.85 \pm 2.08 vs. $4.56 \pm 1.94, \mathrm{P}=0.148)$ and lead impedance $(616.76 \pm 148.89$ vs. $493.09 \pm 118.85, \mathrm{P}=0.108)$ were remain relatively stable during followup duration.

\section{Discussion}

we proved that HBP and LBBP upgrades can improve the heart function, thus reverse the left ventricular remodeling in patients with PICM. Furthermore, we first demonstrated the improvement could also be found in patients with PICM and permanent AF.

\section{His-purkinje system pacing improves cardiac performances}

The chronic RVP might induce inter- and intraventricular dyssynchrony, which is detrimental to left ventricular function and associated with heart failure and increased mortality [10]. The incidence of PICM was relatively high [11]. However, about $30 \%$ of patients were no response to CRT [12]. A series of publications have suggested that HBP could provide favorable clinical results in patients with CRT indications [9, 13]. Notably, Sharma et al. indicated that HBP showed a positive clinical response in CRT nonresponsive patients [5]. The His-SYNC trial [14] was the first prospective, randomized to compared the performance of HBP and conventional CRT. The narrower QRS was observed in HBP group but the improvement of cardiac function and survival were no different between the CRT group and HBP group. Consistently with the studies of Vijayaraman and Sung, our findings also demonstrated the clinical benefits of HBP upgrades in PICM patients $[8,11]$. Furthermore, we also found that the ratio response to HBP/LBBP upgrade were relatively high $(31 / 34,89.66 \%)$ in patients with PICM. Furthermore, 12 patients meet replacement indicators before the upgrade procedure, which indicated that HBP is still efficient and should be a promising choice even in the patients with long-term RVP.

\section{His-purkinje system pacing improves cardiac performances in patients with AF}

To date, the majority of randomized controlled trials and recommendation of current guidelines for CRT upgrades focus on the patients with PICM and sinus rhythm. The biventricular pacing upgrade is still not the optimal recommendation (IIb) in recent guidelines. The efficacy of CRT upgrade was unfavorable in patients with AF due to the potential reduces of biventricular pacing percentage [16,17]. Quite different with those study, In our study, $62 \%$ patients were AF and they had similar clinical responses with the patients with sinus rhythm. For the patients dependent on ventricular pacing, HBP would be the optimal choice? We still need more evidence in future studies.

\section{Current knowledge of upgrade procedure}

HBP has been thought to be associated with several limitations, such as higher capture thresholds, especially in the setting of BBB or infranodal block; lower R-wave amplitudes; and increased risk for lead revisions from late threshold increase. Indeed, as abovementioned, survival prognosis of HBP application was still unclear due to lacking randomized evidence. Thus, HBP has not been widely generalized in clinical practice and is a class IIa indication in the latest guidelines for the management of bradyarrhythmia [15]. In our study, we found that the distal HBP and LBBP were helpful for the better capture thresholds and R-wave. The distal HBP and LBBP pacing, fixed in the septal myocardium, could provide ideal capture thresholds, high R-wave amplitudes. The key point for successful pacing is bypass conduction blocks area of distal his bundle or proximal left bundle. We were able to achieve distal HBP /LBBP in 34 of 36 patients with infranodal AV block in this study, which proved the possibility to achieve high success rates of physiologic pacing in patients dependent to ventricular pacing. The long-term effects of his-purkinje pacing on the septal contractile stress need to be further evaluated.

\section{Safety of upgrades for patients with PICM}

Vijayaraman et al demonstrated that the HBP threshold could remain relatively stable after 2 years of follow- 
up [8]. A recent publication confirmed this finding in a larger sample and showed over $90 \%$ of patients have a capture threshold less than $2.5 \mathrm{~V}$ @ 1ms after a median follow-up of 3 years [16]. In 2 patients failed with HBP, we were not able to capture the distal His-Purkinje system even at high output. In the prior reported by Vijayaraman et al [9], the successful rate of HBP upgrade was $95 \%(57 / 60)$ in western population with AV block. These finding suggest that the progression of conduction dysfunction was extremely rare even in PICM patients. These results extend the long-term data about the threshold for HBP. According to our results, the threshold for HBP was not significant higher after the more than 12-month follow-up period. Device malfunction, lead perforation and embolization were not found during follow-up. The technical challenges in HBP are highly dependent on the operators' experience [17-19].

Although the results presented are favorable, there are some limitations in the present study. First, HBP was conducted by experienced implanters, and there were high success rates in our study along with potential patient selection bias. Second, the present study focused on PICM patients with reduced LVEFs. PICM patients with preserved LVEFs may have unique pathological progress and deserve more clinical assessment. Third, long-term pacing performance of the his pacing lead and the potential risks of lead extractions need further careful evaluation.

\section{Conclusions}

HBP and LBBP upgrades improved the heart performance and reversed the left ventricular remodeling in patients suffering from PICM with or without AF significantly, and it should be actively consideration in patients with PICM.

\section{Reference}

1. Sweeney MO, Hellkamp AS, Ellenbogen KA, Greenspon AJ, Freedman RA, Lee KL, et al. Adverse effect of ventricular pacing on heart failure and atrial fibrillation among patients with normal baseline QRS duration in a clinical trial of pacemaker therapy for sinus node dysfunction. Circulation. 2003;107(23):2932-7.

2. Wilkoff BL, Cook JR, Epstein AE, Greene HL, Hallstrom AP, Hsia H, et al. Dual-chamber pacing or ventricular backup pacing in patients with an implantable defibrillator: the Dual Chamber and VVI Implantable Defibrillator (DAVID) Trial. JAMA. 2002;288(24):3115-23.

3. Khurshid S, Epstein AE, Verdino RJ, Lin D, Goldberg LR, Marchlinski FE, et al. Incidence and predictors of right ventricular pacing-induced cardiomyopathy. Heart Rhythm. 2014;11(9):1619-25.

4. Ponikowski P, Voors AA, Anker SD, Bueno H, Cleland JGF, Coats AJS, et al. 2016 ESC Guidelines for the diagnosis and treatment of acute and chronic heart failure: The Task Force for the diagnosis and treatment of acute and chronic heart failure of the European Society of Cardiology (ESC)Developed with the special contribution of the Heart Failure Association (HFA) of the ESC. Eur Heart J. 2016;37(27):2129-200.

5. Sharma PS, Dandamudi G, Herweg B, Wilson D, Singh R, Naperkowski A, et al. Permanent His-bundle pacing as an alternative to biventricular pacing for cardiac resynchronization therapy: A multicenter experience. Heart Rhythm. 2018;15(3):413-20.

6. Occhetta E, Bortnik M, Magnani A, Francalacci G, Piccinino C, Plebani L, et al. Prevention of ventricular desynchronization by permanent para-Hisian pacing after atrioventricular node ablation in chronic atrial fibrillation: a crossover, blinded, randomized study versus apical right ventricular pacing. J Am Coll Cardiol. 2006;47(10):1938-45.

7. Sharma PS, Dandamudi G, Naperkowski A, Oren JW, Storm RH, Ellenbogen KA, et al. Permanent Hisbundle pacing is feasible, safe, and superior to right ventricular pacing in routine clinical practice. Heart Rhythm. 2015;12(2):305-12.

8. Vijayaraman P, Herweg B, Dandamudi G, Mittal S, Bhatt AG, Marcantoni L, et al. Outcomes of His-bundle pacing upgrade after long-term right ventricular pacing and/or pacing-induced cardiomyopathy: Insights into disease progression. Heart Rhythm. 2019. 
9. Shan P, Su L, Zhou X, Wu S, Xu L, Xiao F, et al. Beneficial effects of upgrading to His bundle pacing in chronically paced patients with left ventricular ejection fraction $<50$. Heart Rhythm. 2018;15(3):405-12.

10. Abdelrahman M, Subzposh FA, Beer D, Durr B, Naperkowski A, Sun H, et al. Clinical Outcomes of His Bundle Pacing Compared to Right Ventricular Pacing. J Am Coll Cardiol. 2018;71(20):2319-30.

11. Cho SW, Gwag HB, Hwang JK, Chun KJ, Park KM, On YK, et al. Clinical features, predictors, and long-term prognosis of pacing-induced cardiomyopathy. Eur J Heart Fail. 2019;21(5):643-51.

12. Brugada J, Delnoy PP, Brachmann J, Reynolds D, Padeletti L, Noelker G, et al. Contractility sensorguided optimization of cardiac resynchronization therapy: results from the RESPOND-CRT trial. Eur Heart J. 2017;38(10):730-8.

13. Yu Z, Chen R, Su Y, Chen X, Qin S, Li M, et al. Integrative and quantitive evaluation of the efficacy of his bundle related pacing in comparison with conventional right ventricular pacing: a meta-analysis. BMC Cardiovasc Disord. 2017;17(1):221.

14. Upadhyay GA, Vijayaraman P, Nayak HM, Verma N, Dandamudi G, Sharma PS, et al. His Corrective Pacing or Biventricular Pacing for Cardiac Resynchronization in Heart Failure. J Am Coll Cardiol. 2019;74(1):157-9.

15. Kusumoto FM, Schoenfeld MH, Barrett C, Edgerton JR, Ellenbogen KA, Gold MR, et al. 2018 ACC/AHA/HRS Guideline on the Evaluation and Management of Patients With Bradycardia and Cardiac Conduction Delay: A Report of the American College of Cardiology/American Heart Association Task Force on Clinical Practice Guidelines and the Heart Rhythm Society. Circulation. 2019;140(8):e382-e482.

16. Zanon F, Abdelrahman M, Marcantoni L, Naperkowski A, Subzposh FA, Pastore G, et al. Long term performance and safety of His bundle pacing: A multicenter experience. J Cardiovasc Electrophysiol. 2019;30(9):1594-601.

17. Dandamudi G, Vijayaraman P. The Complexity of the His Bundle: Understanding Its Anatomy and Physiology through the Lens of the Past and the Present. Pacing Clin Electrophysiol. 2016;39(12):1294-7.

18. Qian Z, Zou F, Wang Y, Qiu Y, Chen X, Jiang H, et al. Permanent His bundle pacing in heart failure patients: A systematic review and meta-analysis. Pacing Clin Electrophysiol. 2019;42(2):139-45.

19. Vijayaraman P, Naperkowski A, Subzposh FA, Abdelrahman M, Sharma PS, Oren JW, et al. Permanent His-bundle pacing: Long-term lead performance and clinical outcomes. Heart Rhythm. 2018;15(5):696-702.

Table 1 Baseline characteristics of patients upgrade to HBP

\begin{tabular}{ll}
\hline & PICM patients $(\mathrm{n}=34)$ \\
Age (years) & $69.69 \pm 13.75$ \\
male (n, \%) & $22(64.7 \%)$ \\
Diabetes mellitus (n, \%) & $6(17.6 \%)$ \\
Coronary heart disease (n, \%) & $5(14.7 \%)$ \\
Hypertension (n, \%) & $23(67.6 \%)$ \\
Renal dysfunction (n, \%) & $3(8.8 \%)$ \\
CLBBB (n, \%) & $3(8.8 \%)$ \\
Lipid-regulating drugs (n, \%) & $9(26.5 \%)$ \\
Anticoagulants (n, \%) & $17(50.0 \%$ \\
ACEI/ARB/ARNI (n, \%) & $12(35.3 \%)$ \\
$\beta$-blockers (n, \%) & $29(85.3 \%)$ \\
ARNI (n, \%) & $6(17.6 \%)$ \\
Diuretics (n, \%) & $29(85.3 \%)$ \\
Digoxin (n, \%) & $7(20.6 \%)$ \\
LVEF before RVP (\%) & $51.77 \pm 8.19$
\end{tabular}




$\begin{array}{ll}\text { LVEF before HBP (\%) } & 33.76 \pm 7.54 \\ \text { LVEDD before RVP (mm) } & 54.83 \pm 7.35 \\ \text { LVEDD before HBP (mm) } & 59.29 \pm 7.74 \\ \text { NYHA classification } & 2.55 \pm 0.65 \\ \text { QRS duration (ms) } & 184.2 \pm 23.67 \\ \text { VP percentage (\%) } & 90.54 \pm 24.01 \%\end{array}$

$C L B B B$ : complete left bundle branch block; $A C E I$ :angiotensin converting enzyme inhibitors; $A R B$ angiotensin receptor blocker; $A R N I$ : angiotensin receptor neprilysin inhibitors; $L V E D D$ left ventricular end-diastolic diameter; $L V E F$ left ventricular ejection fraction; $V P$ : ventricular pacing.

Table 2. Clinical outcomes of upgrades

\begin{tabular}{llll}
\hline & baseline $(\mathrm{n}=34)$ & final follow-up $(\mathrm{n}=34)$ & $P$ value \\
QRSd (ms) & $184.2 \pm 23.67$ & $120.5 \pm 16.67$ & 0.000 \\
NYHA classification & $2.55 \pm 0.91$ & $2.00 \pm 0.76$ & 0.000 \\
final LA size & $47.44 \pm 7.14$ & $45.56 \pm 7.78$ & 0.010 \\
LVEDD (mm) & $59.29 \pm 7.74$ & $53.91 \pm 5.92$ & 0.000 \\
MR & 10 & 6 & 0.219 \\
TR & 10 & 8 & 0.727 \\
LVEF $(\%)$ & $33.76 \pm 7.54$ & $40.41 \pm 9.06$ & 0.000 \\
\hline
\end{tabular}

$L A$ left atrium, $L V E D D$ left ventricular end-diastolic diameter, $L V E F$ left ventricular ejection fraction, $M R$ mitral regurgitation, $T R$ tricuspid regurgitation.

Table3. Clinical outcomes in patients with or without AF

\begin{tabular}{llll}
\hline & AF group $(\mathbf{n = 2 1})$ & $\begin{array}{l}\text { Sinus rhythm group } \\
(\mathbf{n = 1 3})\end{array}$ & $\mathbf{P}$ \\
Age & $66.62 \pm 14.59$ & $73 \pm 10.21$ & \\
Gender (male, \%) & $15(65.2 \%)$ & $8(53.3 \%)$ & \\
VP percent (\%) & $85.48 \pm 29.95$ & $98.32 \pm 3.02$ & 0.135 \\
initial QRSd (ms) & $185.35 \pm 21.79$ & $182.08 \pm 28.98$ & 0.731 \\
final QRSd (ms) & $119.35 \pm 16.26^{*}$ & $122.83 \pm 18.95^{*}$ & 0.600 \\
initial NYHA & $2.69 \pm 0.91$ & $2.33 \pm 0.67$ & 0.377 \\
classification & & & \\
final NYHA & $2.06 \pm 0.75^{*}$ & $1.77 \pm 0.63^{*}$ & 0.261 \\
classification & & & \\
initial LA size (mm) & $51.05 \pm 6.45$ & $41.62 \pm 3.36$ & 0.000 \\
final LA size (mm) & $49.71 \pm 6.83$ & $38.85 \pm 3.13^{*}$ & 0.000 \\
initial LVEDD (mm) & $59.67 \pm 7.74$ & $58.69 \pm 8.03$ & 0.727 \\
final LVEDD (mm) & $54.05 \pm 5.88^{*}$ & $53.69 \pm 6.22^{*}$ & 0.868 \\
initial LVEF (\%) & $34.71 \pm 6.38$ & $32.23 \pm 9.18$ & 0.358 \\
final LVEF (\%) & $40.57 \pm 9.38^{*}$ & $40.15 \pm 8.90^{*}$ & 0.898 \\
\hline
\end{tabular}

$L A$ left atrium, $L V E D D$ left ventricular end-diastolic diameter, $L V E F$ left ventricular ejection fraction, $M R$ mitral regurgitation, $T R$ tricuspid regurgitation.

$* \mathrm{P}<0.05$ comparison between before HBP procedure and last follow-up

Table 4. Lead outcomes during the operation and final follow-up 


\begin{tabular}{llll}
\hline & baseline & final follow-up & $P$ value \\
VP percentage (\%) & $81.67 \pm 32.16 \%$ & $92.79 \pm 19.15 \%$ & 0.124 \\
Threshold (V@0.4ms) & $1.18 \pm 0.76$ & $1.26 \pm 0.91$ & 0.581 \\
Threshold in AF subgroup & $1.07 \pm 0.70$ & $1.15 \pm 0.70$ & 0.582 \\
Threshold in Non-AF subgroup & $1.34 \pm 0.84$ & $1.44 \pm 1.19$ & 0.788 \\
Impedance $(\Omega)$ & $616.76 \pm 148.89$ & $493.09 \pm 118.85$ & 0.108 \\
Sensed R wave $(\mathrm{mV})$ & $4.85 \pm 2.08$ & $4.56 \pm 1.94$ & 0.148 \\
\hline
\end{tabular}

$V P$ ventricular pacing
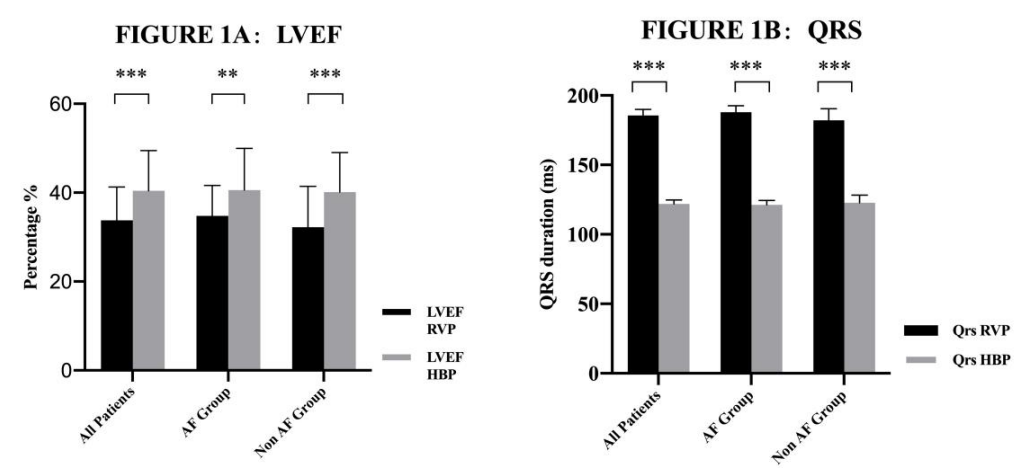

FIGURE 1C: LVEDD

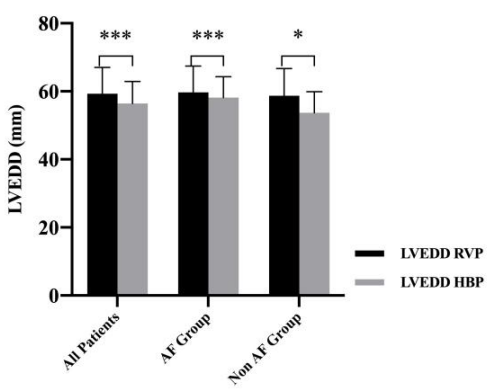

FIGURE 1D: Threshold of his bundle lead

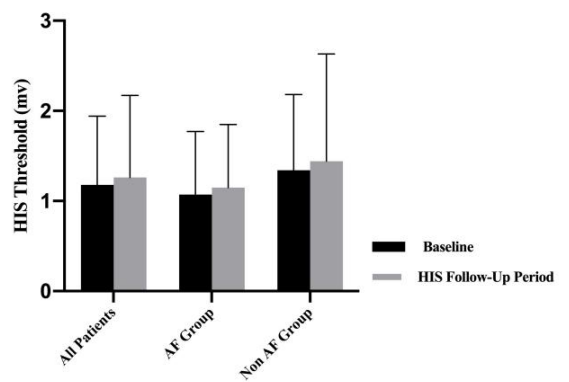

Figure 1A Comparison of changes in LVEF before and after HBP upgrad,

Figure 1B Comparison of changes in QRSd between RV and HBP,

Figure 1C Comparison of changes in LVEDD between RV and HBP,

Figure 1D Comparison of changes in threshold of HBP between post-operative time and follow-up period 